Medische Bibliotheek, Erasmus MC Rotterdam. Tel: 010-4087782

Gegevens aanvrager / verzendadres

Ref:

\begin{tabular}{|l|}
\hline Heiman F.L. Wertheim \\
Medical Microbiology \\
L348 \\
Erasmus MC \\
Dr Molewaterplein 40 \\
3015 GD Rotterdam \\
\hline
\end{tabular}

Tel: $\quad 0104633510$

Fax:

E-mail: h.wertheim @erasmusmc.nl

Budget: 2230

Aanvraag kopie / Boek te leen

ISSN p/e: 0934-9723/1435-4373

MBSN:

Titel: $\quad$ Eur J Clin Microbiol Infect Dis

Jaar: $\quad 1994$

Volume: 13

Aflevering: 1

Auteur: $\quad$ Valls V, GÄ $\tilde{A}^{3}$ mez-Herruz P, Gonz $\tilde{A}_{j}$ lez-Palacios R, Cua

Artikel: Long-term efficacy of a program to control methicillin

Pagina's: $\quad 90-5$

PMID: $\quad 8168570$

Opmerking:

Subject: Normal electronic delivery

Date: $\quad$ Thu, 14 Jul 2005 10:37:32

From: $\quad$ h.wertheim @erasmusmc.nl

Nota adres (indien anders dan verzendadres)

Heiman F.L. Wertheim Erasmus MC

Medical Microbiology

Dr Molewaterplein 40

3015 GD Rotterdam

Aktie: Levering uit MBTSZ collectie 


\title{
Long-Term Efficacy of a Program to Control Methicillin-Resistant Staphylococcus aureus
}

\author{
V. Valls ${ }^{1,4 *}$, P. Gómez-Herruz ${ }^{2}$, R. González-Palacios ${ }^{2}$, J.A. Cuadros ${ }^{2}$, J.P. Romanyk ${ }^{2}$, J. Ena ${ }^{3}$
}

\begin{abstract}
The long-term efficacy of a program to control methicillin-resistant Staphylococcus aureus (MRSA) was evaluated in a 350-bed university hospital. Three periods were monitored: pre-epidemic (January 1989-November 1989), outbreak (December 1989-June 1990) and control program (July 1990-December 1992) periods. Control measures included cohort isolation, patient care measures and therapy (oral cotrimoxazole plus fusidic acid ointment) of MRSA carriage in patients, roommates and personnel. A total of $117 \mathrm{MRSA}$-infected patients were detected. For each period respectively, MRSA incidence (number of cases per 1,000 patient-days) was 3.2, 8.2 and 2.0 in the intensive care unit (ICU) and $0.08,0.23$ and 0.26 in the general wards. During the outbreak there was a 2.7-fold overall increase of baseline MRSA incidence $(p<0.02)$. The crude mortality was $68 \%$ and the attributable mortality was estimated to be $50 \%$. The program was estimated to have prevented $76 \%$ (C195 28-91, p $<0.0001$ ) of expected MRSA cases and $85 \%$ (CI95 62-94, p < 0.0001) of expected fatalities due to MRSA in the ICU, but it had no significant effect in the general wards. The program did not control vancomycin consumption.
\end{abstract}

During the last decade outbreaks due to methicillin-resistant Staphylococcus aureus (MRSA) have been reported with increasing frequency (1$5)$. Although some authors argue that MRSA infections create little impact in the hospital (6), others have demonstrated the ability of these microorganisms to cause severe infection (7). In fact, in some institutions MRSA has become the leading cause of nosocomial bloodstream infections (8). This has led to changes in routine empirical and culture-directed therapies, resulting in an increased consumption of vancomycin. Infection control programs ameliorated the impact of MRSA epidemics, even in institutions with limited resources $(1,2)$. However, the benefits of major programs to control MRSA are still debated (9).

In June 1990 we detected an MRSA outbreak that mainly affected patients in the intensive care unit. A series of approaches to control the infections was implemented. The present study evalu-

\footnotetext{
${ }^{1}$ Servicio de Medicina Preventiva, ${ }^{2}$ Servicio de Microbiología Clínica and ${ }^{3}$ Servicio de Medicina Interna, Hospital Universitario Príncipe de Asturias, Alcalá de Henares, 28805 Madrid, Spain.

${ }^{4}$ Servicio de Medicina Preventiva, Hospital General SVS de Alicante, Maestro Alonso 109, 03010 Alicante, Spain.
}

ates the efficacy of the program based on MRSA incidence, crude and attributable mortality from MRSA infections and vancomycin consumption before and after the control measures were implemented.

\section{Materials and Methods}

Hospital. Hospital Principe de Asturias is a 350-bed teaching institution located in the northeastern area of Madrid, Spain, with approximately 1,200 admissions per month. The hospital opened in November 1987. The medical intensive care unit contains seven individual patient cubicles open to the nursing facilities and one isolation room. The post-surgical care unit is a large open area with 14 beds. The medical and surgical departments have two-bed rooms in units of 15 rooms. There are no special units for immunocompromised or burn patients. Hospital-wide surveillance of multiple-antibiotic-resistant organisms began in January 1988. Vancomycin consumption is routinely monitored by the Department of Pharmacy.

Microbiological Methods. Standard methods used to identify Staphylococcus aureus included the Gram stain, colonial and microscopic morphology and the catalase and tube coagulase tests (10). Susceptibility testing was performed by the microtiter dilution technique using an automated method (Pasco, USA). Antibiotics tested were penicillin, ampicillin, amoxicillin plus clavulanic 
acid, oxacillin, cefazolin, cefotaxime, imipenem, gentamicin, tobramycin, clindamycin, erythromycin, rifampin, norfloxacin, ciprofloxacin, trimethoprim-sulfamethoxazole, fosfomycin and vancomycin. Resistance to methicillin was defined by an oxacillin minimal inhibitory concentration of $6.0 \mu \mathrm{g} / \mathrm{ml}$ or greater. Determination of susceptibility to mupirocin and fusidic acid was performed by disk diffusion tests (11). The epidemic strain was identified by antibiogram and phage typing (12). Phage typing was done at the National Reference Center (Centro Nacional de Microbiologia, Virologia e Immunologia Sanitarias de Majadahonda, Madrid, Spain).

Definitions and Rates. We reviewed the charts of all patients with MRSA isolation in clinical samples. A patient was considered to have an infection due to MRSA based on the Centers for Discase Control definitions for nosocomial infection (13). Otherwise, isolations of MRSA from clinical samples were considered to be colonizations. MRSA infection rates were determined by the number of patients infecled with MRSA per 1,000 patient-days. All deaths in patients with MRSA were noted. Death was considered to be related to an MRSA infection when no other cause of death could be identified and, at the time of fatality, the patient had symptoms or signs of infection caused by MRSA or a clinical complication of the infection. MRSA crude mortality rates were determined by the overall number of deaths observed in MRSA-infected patients per 1,000 patient-days. MRSA attributable mortality was estimated by the number of deaths thought to be related to an MRSA infection per 1,000 patient-days. The number of MRSA cases and fatalities prevented was estimated by means of the etiologic fraction parameter, which represents the expected reduction in disease load following implementation of the program (14). To estimate the etiologic fraction, the difference between incidence rate of the program period and the epidemic period was divided by the incidence rate of the epidemic pcriod. Vancomycin consumption rates were defined by the number of grams of vancomycin used per 1,000 patient-days.

MRSA Control Program. The program to control MRSA was based mainly on the measures recommended by the Hospital Infection Socicty and the British Sociely for Antimicrobial Chemotherapy (15). The infection control procedures used are summarized in Table 1 .

Statistical Analysis. Comparisons of incidence rates were performed by chi-square for patient-days estimations (16). Ninety-five percent confidence intervals (CI95) were calculated. For etiologic fractions, the Cl95s were estimated using the approximate standard error derived from the Taylor series method (17). Two-tailed $p$ values $<0.05$ were considered significant.

\section{Results}

Pre-Epidemic and Oulbreak Period. From January 1989 to June 1990, before the control program began, a single strain of MRSA caused 49 infections in 43 patients. The epidemic strain was resistant to methicillin, ampicillin, amoxicillin plus clavulanic acid, aminoglycosides, macrolidelincosamine group, rifampin, quinolones and imipenem. It was susceptible to vancomycin, trimethoprim-sulfamethoxazole, fosfomycin, fusidic acid and mupirocin. Organisms were lysed by phages belonging to group III.

Fifteen patients were diagnosed during the preepidemic period (January 1989 to November

Table 1: Measures used to control MRSA.

\begin{tabular}{|c|c|c|c|}
\hline Patient care & $\begin{array}{l}\text { Single room and coho } \\
\text { Gowns, gloves and m } \\
\text { Chlorhexidine soap I }\end{array}$ & $\begin{array}{l}\text { ation for infected/cc } \\
\text { or every person cntc } \\
\text { sonnel hand washing }\end{array}$ & $\begin{array}{l}\text { patients; } \\
\text { room; }\end{array}$ \\
\hline \multirow{4}{*}{$\begin{array}{l}\text { Screening for } \\
\text { calriers }\end{array}$} & Group & Siles sampled & When sampled \\
\hline & $\begin{array}{l}\text { MRSA infected or } \\
\text { colonized patients }\end{array}$ & $\begin{array}{l}\text { nose, axilla, } \\
\text { cutancous lesions, } \\
\text { infection sites }\end{array}$ & weekly until discharge \\
\hline & $\begin{array}{l}\text { Roommates of } \\
\text { MRSA infected or } \\
\text { colonized patients }\end{array}$ & $\begin{array}{l}\text { nose, axilla, } \\
\text { cutaneous lesions, } \\
\text { infection sites }\end{array}$ & $\begin{array}{l}\text { baseline and weckly until } \\
\text { discharge, if isolation was } \\
\text { not feasible }\end{array}$ \\
\hline & Health care workers & $\begin{array}{l}\text { nosc, hands, } \\
\text { cutaneous lesions }\end{array}$ & $\begin{array}{l}\text { if } 3 \text { or more cases of } \\
\text { MRSA infection were } \\
\text { detected in the same ward } \\
\text { within a 15-day period }\end{array}$ \\
\hline
\end{tabular}

Treatment of Daily bath with chlorhexidine soap;

carriers Topical fusidic acid (t.i.d., 5 days) in nose and cutancous lesions;

Oral trimethoprim-sulfamethoxazolc (160-800 mg b.i.d., 5 days). 
Table 2: Crude data and rates of MRSA-infected patients and vancomycin consumption.

\begin{tabular}{|c|c|c|c|}
\hline & $\begin{array}{l}\text { Pre-epidemic period } \\
\text { (Jan. 1989-Nov. 1989) }\end{array}$ & $\begin{array}{c}\text { Outbreak period } \\
\text { (Dec. 1989-June 1990) }\end{array}$ & $\begin{array}{c}\text { Program period } \\
\text { (July 1990-Dec. 1992) }\end{array}$ \\
\hline \multicolumn{4}{|l|}{ Intensive care unit } \\
\hline No. of MRSA-infected patients & 9 & 15 & 12 \\
\hline MRSA crude mortality & $55.5 \%$ & $86.6 \%$ & $58.3 \%$ \\
\hline MRSA attributable mortality & $33.3 \%$ & $66.6 \%$ & $41.6 \%$ \\
\hline Vancomycin consumption & $215 \mathrm{~g}$ & $191 \mathrm{~g}$ & $646 \mathrm{~g}$ \\
\hline No. of patient-days & 2,794 & 1,833 & 5,997 \\
\hline $\begin{array}{l}\text { MRSA infection rate } \\
\text { (no./1000 patient-days) }\end{array}$ & 3.2 & $8.2(\mathrm{p}<0.0001)^{\mathrm{a}}$ & $2.0(\mathrm{p}<0.0001)^{b}$ \\
\hline $\begin{array}{l}\text { MRSA crude mortality rate } \\
\text { (no./1000 patient-days) }\end{array}$ & 1.78 & $7.09(\mathrm{p}=0.0050)^{\mathrm{a}}$ & $1.17(\mathrm{p}<0.0001)^{\mathrm{b}}$ \\
\hline $\begin{array}{l}\text { MRSA attributable mortality ratc } \\
\text { (no./1000 patient-days) }\end{array}$ & 1.07 & $5.45(\mathrm{p}=0.0067)^{\mathrm{a}}$ & $0.83(p<0.0001)^{b}$ \\
\hline $\begin{array}{l}\text { Vancomycin consumption rate } \\
\text { (grams } / 1000 \text { patient-days) }\end{array}$ & 76.9 & $104.2(\mathrm{p}=0.0016)^{\mathrm{a}}$ & $107.7(p=0.34)^{b}$ \\
\hline \multicolumn{4}{|l|}{ Gencral wards } \\
\hline No. of MRSA-infected patients & 6 & 13 & 62 \\
\hline MRSA crude mortality & $50.0 \%$ & $46.2 \%$ & $17.7 \%$ \\
\hline MRSA attributable mortality & $33.3 \%$ & $30.8 \%$ & $14.5 \%$ \\
\hline Vancomycin consumption & $536 \mathrm{~g}$ & $928 \mathrm{~g}$ & $3,682 \mathrm{~g}$ \\
\hline No. of patient-days & 70,752 & 57,123 & 238,703 \\
\hline $\begin{array}{l}\text { MRSA infection ratc } \\
\text { (no./1000 patient-days) }\end{array}$ & 0.08 & $0.23(p=0.0180)^{a}$ & $0.26(p=0.3300)^{b}$ \\
\hline $\begin{array}{l}\text { MRSA crude mortality rate } \\
\text { (no./1000 patient-days) }\end{array}$ & 0.04 & $0.11(p=0.1840)^{a}$ & $0.05(\mathrm{p}=0.0870)^{\mathrm{b}}$ \\
\hline $\begin{array}{l}\text { MRSA attributable mortality rate } \\
\text { (no./1000 patient-days) }\end{array}$ & 0.03 & $0.07(\mathrm{p}=0.2880)^{\mathrm{a}}$ & $0.04(p=0.2753)^{b}$ \\
\hline $\begin{array}{l}\text { Vancomycin consumption rate } \\
\text { (grams } / 1000 \text { patient-days) }\end{array}$ & 7.57 & $16.2(\mathrm{p}<0.0001)^{\mathrm{a}}$ & $15.42(\mathrm{p}=0.0790)^{\mathrm{b}}$ \\
\hline
\end{tabular}

${ }^{a} \mathrm{p}$ value of the outbreak period compared with the pre-epidemic period.

$b_{p}$ value of the program period compared with the outbreak period.

1989) and 28 during the outbreak period (December 1989 to June 1990). In both periods the MRSA incidence rate was 40 -fold greater in the intensive care unit than in the general wards ( $p<0.0001)$. The sources of infections were intravascular catheter-related $(\mathrm{n}=13)$, respiratory tract $(n=10)$, surgical wound $(n=6)$, urinary tract $(n=5)$ and miscellaneous $(n=9)$. There were six cases of primary bacteremia and 19 cases of secondary bacteremia. Twenty-seven patients with MRSA infection died, resulting in a crude mortality of $63 \%$. In 19 patients death was thought to be related to the infection, resulting in an estimated attributable mortality of $44 \%$. In the intensive care unit MRSA crude and attributable mortality within the outbreak period reached $87 \%$ and $67 \%$, respectively.

Compared with the pre-epidemic period, in the outbreak period there was a 2.7 -fold increase in the MRSA incidence rate in both the intensive care unit and the general wards (Table 2). Differ- ences in MRSA incidence rates between the preepidemic and the outbreak period were 5 per 1,000 patient-days (CI95 0.36-9.64, $\mathrm{p}<0.0001$ ) in the intensive care unit and 0.14 per 1,000 patientdays (CI95 0.07-0.21, $\mathrm{p}=0.0180$ ) in the general wards. In the intensive care unit MRSA crude and estimated attributable mortality rates increased 4-fold and 5.1-fold, respectively. Crude and estimated attributable mortality rate differences between the outbreak period and the pre-epidemic period were 5.3 per 1,000 patient-days (CI95 1.6$9.0, \mathrm{p}=0.0050)$ and 4.4 per 1,000 patient-days (CI95 0.5-8.3, p = 0.0067), respectively. Vancomycin consumption increased 1.4-fold $(p=0.0016)$. In the general wards MRSA crude and estimated attributable mortality rates increased 2.7-fold $(p=0.0180)$ and 2.3-fold $(p=0.2880)$, respectively. Vancomycin consumption increased 2.1-fold ( $\mathrm{p}<0.0001)$ (Table 2).

MRSA Control Program Period. According to the measures described in Table 1, MRSA-in- 


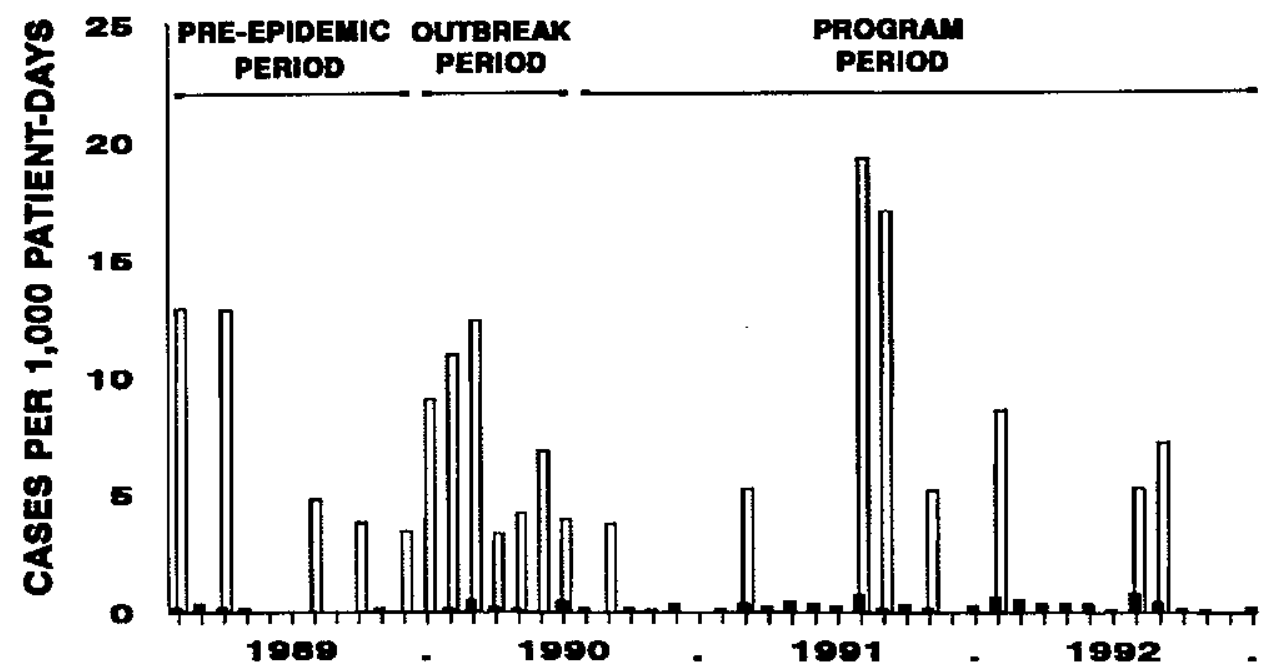

Figure 1: Evolution of cases of MRSA-infected patients (cases per 1,000 patient-days) in the intensive care unit (hollow bars) and in the general wards (black bars).

fected patients, their roommates and health care workers in the intensive care unit were screened for MRSA carriage during the program period. Nasal carriage was detected in 37 of $64(58 \%)$ MRSA-infected patients screened. Weekly microbiologic surveillance for MRSA carriage was maintained among MRSA-infected patients even if baseline screening cultures were negative. Five of $27(18.5 \%)$ MRSA-infected patients with negative baseline screening cultures became carriers during their admission.

Among roommates of MRSA-infected patients in the intensive care unit and general wards, nine of $56(16 \%)$ and 15 of $62(24 \%)$ were nasal carriers $(\mathrm{p}=0.38)$, respectively. In July 1990 , four of 52 $(7.7 \%)$ health care workers were nasal carriers, whereas in July 1991, when a second outbreak was recognized, only one of $52(2 \%)$ health care workers was found to be a nasal carrier $(p=0.36)$. Cutaneous carriage was detected in $30 \%$ of MRSA-infected patients, in $9 \%$ of MRSA roommates in the intensive care unit, in $10 \%$ of MRSA roommates in the general wards and in $1.9 \%$ of health care workers. Cutaneous carriage was always associated with nasal carriage.

Microbiologic surveillance was performed in 52 nasal carriers. Eradication of MRSA carriage, defined by two consecutive negative swabs (days 5 and 10 after treatment), was achieved in 42 persons $(81 \%)$. However, seven of $31(23 \%)$ persons relapsed within a medium follow-up of 38 days (limits: 17-101 days). Organisms isolated showed the same antibiogram as the initial strain. Relapse was detected after a medium time of 52 days (limits: 17-94 days).

From the implementation of the program in July 1990 through June 1991, there was a significant reduction of MRSA-infected patients in the intensive care unit. The MRSA incidence rate dropped from 8.2 to 0.7 cases per 1,000 patientdays $(p<0.0001)$ (Figure 1). However, a second outbreak was recognized in July 1991. Five patients ( 18 cases per 1,000 patient-days) were affected in the intensive care unit within two months. Despite these cases, the overall MRSA incidence rate within the control program period (July 1990-December 1992) decreased 4.1-fold in the intensive care unit compared with the outbreak period.

The MRSA infection rate difference was 6.2 per 1,000 patient-days (CI95 1.9-10.5, $\mathrm{p}<0.0001$ ). There was also a significant reduction of both the crude and the estimated attributable mortality rates in the intensive care unit (Table 2). Crude mortality decreased from 7.09 to 1.17 per 1,000 patient-days and estimated attributable mortality from 5.45 to 0.83 per 1,000 patient-days. Thus, there were rate differences of 5.9 per 1,000 patient-days (CI952.9-8.5, p < 0.0001) and 4.6 per 1,000 patient-days (CI95 2.3-6.9, $\mathrm{p}<0.0001$ ), respectively, compared with the outbreak period. Vancomycin consumption remained unchanged during the program period. There were no changes in MRSA incidence, mortality rates or vancomycin consumption in the general wards (Table 2). 
The estimated proportion of MRSA cases prevented in the intensive care unit was $76 \%$ (CI95 $28-91, \mathrm{p}<0.0001$ ), and the estimated proportion of deaths prevented was $85 \%$ (CI95 62-94, $\mathrm{p}<0.0001$ ). The program had no significant effect on vancomycin consumption or on MRSA rates in the general wards, although the proportion of expected deaths prevented was estimated to be $45 \%$.

\section{Discussion}

From December 1989 to June 1990 a single strain of MRSA caused severe infections in 28 patients in our hospital. Among patients with bacteremia, over $50 \%$ died. Subsequently, an infection control program was implemented to control this outbreak. Thereafter, MRSA infection rates decreased sharply in the intensive care unit, from 8.2 to 0.7 cases per 1,000 patient-days. The program was estimated to have prevented $76 \%$ of new MRSA cases and $85 \%$ of expected deaths due to MRSA in the intensive care unit. On the general wards of the hospital, MRSA cases continued to occur at a low level of endemicity, with a rate of 0.26 cases per 1,000 patient-days. However, a decrease of expected MRSA mortality of $45 \%$ was estimated. Stringent control measures successfully controlled the outbreak in the intensive care unit and secondarily decreased MRSA mortality. In addition, in areas in which MRSA was endemic there was a reduction in the expected MRSA mortality that might be attributable to earlier detection and therapy of MRSAinfected patients.

Control measures necessary to stop MRSA outbreaks include a system of isolation and identification of MRSA reservoirs. Colonized patients and hospital personnel should be actively identified and undergo nasal decolonization (18-20). Nasal carriage by patients is well known to be associated with increased nosocomial infection rates (21). Furthermore, Murder et al. (22) found that MRSA-colonized patients were four times more likely to acquire staphylococcal infections than those not colonized. On the other hand, the importance of colonization among hospital personnel has been controversial (23). Nevertheless, in some studies nasal decolonization of health care workers contributed toward ending the outbreaks $(24,25)$.

In our institution MRSA nasal colonization was detected in $58 \%$ of MRSA-infected patients.
Therefore, nasal decolonization, in addition to treatment of infection, was required in this group of patients. Asymptomatic MRSA carriage was also found in $20 \%$ of MRSA roommates and in up to $8 \%$ of hospital personnel. Moreover, five of $27(18.5 \%)$ patients with negative baseline screening cultures became MRSA carriers during their hospitalization period. This finding underscores the importance of maintaining microbiological surveillance until patients are discharged.

Nasal mupirocin is considered the best choice for eradication of MRSA carriage (26), but for the period studied, mupirocin was not approved in our country. Classical schemes with topical plus oral antimicrobial agents can also eradicate the carrier status efficiently (27). Oral trimethoprimsulfamethoxazole plus fusidic acid ointment eradicated MRSA in $81 \%$ of carriers found in our hospital. Although no resistance to fusidic acid or trimethoprim-sulfamethoxazole was observed, seven of 31 patients relapsed after a median follow-up of 52 days. Similar and even greater relapse rates have been observed with other therapeutical schemes, including the use of mupirocin (28).

An ancillary measure recommended to control MRSA spread is the identification, on admission, of patients with previous infection or colonization due to MRSA by means of a registration system. This measure was not available in our hospital. Despite the lack of a registration system, the measures implemented in our institution successfully ameliorated the effect of MRSA infections.

An additional aim of this study was to examine the evolution of vancomycin consumption as an indicator of the impact of MRSA infections. Vancomycin usage increased 1.4-fold in the intensive care unit and 2.1-fold in the general wards during the outbreak. After controlling the outbreak, vancomycin consumption remained unchanged. In addition to the expenses incurred during an outbreak, which are related to the extended length of stay and other associated conditions or increased staffing demands (19), excess vancomycin use remained as a consequence. This may have been a result of fear of MRSA infections by concerned physicians. Continuous feedback is needed to keep physicians updated about the distribution and frequency of MRSA infections. Furthermore, automatic stop orders limiting courses of empirical vancomycin to $24-48$ hours might be effective.

In summary, although MRSA outbreaks are difficult to eradicate, stringent control programs can 
ameliorate the morbidity and mortality of MRSA infections. Even after the MRSA outbreak is controlled, vancomycin consumption may remain elevated. Therefore, in institutions with a history of MRSA outbreaks, vancomycin should be included in the category of antibiotics requiring close monitoring.

\section{Acknowledgements}

We thank Dr. R.P. Wenzel for his comments on the previous version of this manuscript.

\section{References}

1. Guiguet M, Rekacewicz C, Leclerq B, Brun Y, Escudier $\mathrm{B}$, Andremont A: Effectiveness of simple measurcs to control an outbreak of nosocomial methicillin-resistant Staphylococcus aureus infections in an intensive care unit. Infection Control and Hospital Epidemiology 1990, 11: 23-26.

2. Murray-Leisure KA, Geib S, Graceley D, Rubin-Slutsky AB, Saxena N, Muller HA, Hamory B: Control of epidemic methicillin-resistant Staphylococcus aureus. Infection Control and Hospital Epidemiology 1990, 11: 343-350.

3. Ransjö U, Malm M, Hambraeus A, Artursson G, Hedlund A: Methicillin-resistant Staphylococcus aureus in two burn units: clinical significance and epidemiological control. Journal of Hospital Infection 1989, 13: 355365.

4. Goetz MB, Mulligan ME, Kwok R, O'Brien H, CabalJers C, García JP: Management and epidemiologic analyses of an outbreak due to methicillin-resistant Staphylococcus aureus. American Journal of Medicine 1992, 92: 607-614.

5. Marples RR, Cooke EM: Current problems with methicillin-resistant Staphylococcus aureus. Journal of Hospital Infection 1988, 11: 381-392.

6. Meers PD, Leong KY: The impact of methicillin- and aminoglycoside-resistant Staphylococcus aureus on the pattern of hospital-acquired infection in an acute hospital. Journal of Hospital Infection 1990, 16: 231239.

7. Peacock JE, Marsik F, Wenzel RP: Methicillin-resistant Staphylococcus aureus: introduction and spread within a hospital. Annals of Internal Medicine 1980, 93: 526-532.

8. Finkelstein R, Markel A, Reinherz G, Hashman N, Merzbach D: The emergence of methicillin-resistant Staphylococcus aureus infections in an Isracli hospital. Journal of Hospital Infection 1989, 14: 55-61.

9. Barrett SP, Mellor JA: Living with EMRSA-1. Journal of Hospital Infection 1990, 15: 103-106.

10. Kloos WE, Jorgensen JH: Staphylococci. In: Lennelte EH, Balows A, Hausler WJ Jr, Shadomy HJ (ed): Manual of clinical microbiology. American Society for Microbiology, Washington DC, 1985 , p. 145-150.

11. National Committec for Clinical Laboratory Standards: Performance standards for antimicrobial disk susceptibility tests. Approved standard M2-A4. NCCLS, Villanova, PA, 1990.
12. Blair JE, Williams REO: Phage typing of staphylococci. Bulletin of the World Health Organization 1961, 24: 771-784.

13. Centers for Disease Control: Outline for surveillance and control of nosocomial infections. Centers for Disease Control, Atlanta, GA, 1970.

14. Schlesseelman JJ: Case-control studies. Oxford University Press, New York, 1982.

15. Working Party Report of the Hospital Infection Society and British Socicty for Antimicrobial Chemotherapy: Revised guidelines for the control of epidemic methicillin-resistant Staphylococcus aureus. Journal of Hospital Infection 1990, 16: 351-377.

16. Rothman KJ: Modern epidemiology. Little Brown, Boston, 1986, p. 155.

17. Kleinbaum DG, Kupper LL, Morgenstem H: Epidemiologic research. Van Nostrand Reinhold, New York, 1982, p. 351.

18. Boyce JM: Should we vigorously try to contain and control methicillin-resistant Staphylococcus aureus? Infection Control and Hospital Epidemiology 1991, 12: 46-54.

19. Rao N, Jacobs S, Joyce L: Cost-effeclive eradication of an outbreak of methicillin-resistant Staphylococcus aureus in a community teaching hospital. Infection Control and Hospital Epidemiology 1988, 9: 255-260.

20. Casewell MW: Epidemiology and control of the "modern" methicillin-resistant Staphylococcus aureus. Journal of Hospital Infection 1986, 7, Supplement A: 1-11.

21. McNeil JJJ, Proudfoot AD, Tosolini FA, Morris P, Booth JM, Doyle AE, Louis WJ: Methicillin-resistant Staphylococcus aureus in an Australian teaching hospital. Journal of Hospital Infection 1984, 5: 18-28.

22. Murder RR, Brennen C, Wagener MM, Vickers RM, Rihs JD, Hancock GA, Yee YC, Miller JM, Yu VL: Methicillin-resistant staphylococcal colonization and infection in a long-term care facility. Annals of Internal Medicine 1991, 114: 107-112.

23. Thompson RL, Cabezudo I, Wenzel RP: Epidemiology of nosocomial infections caused by methicillin-rcsistant Staphylococcus aurets. Annals of Internal.Medicine 1982, 97: 309-317.

24. Hill RL, Duckworth GH, Casewell MW: Elimination of nasal carriage of methicillin-resistant Staphylococcus aureus with mupirocin during a hospital outbrcak. Journal of Antimicrobial Chemotherapy 1988, 22: 377384.

25. Barrett SP: The value of nasal mupirocin in containing an outbreak of methicillin-resistant Staphylococcus aureus in an orthopaedic unit. Journal of Hospital Infection 1990, 15: 137-142.

26. Reagan DR, Docbbeling BN, Pfaller MA, Sheetz Cr, Houston AK, Hollis RJ, Wenzel RP: Elimination of coincident Staphylococcus aureus nasal and hand carriage with intranasal application of mupirocin calcium ointment. Annals of Internal Medicine 1991, 114: 101-106.

27. Mulligan ME, Murray-Leisure KA, Ribner BS, Standiford HC, John JF, Korvick JA, Kauffman CA, Yu VL: Methicillin-resistant Staphylococcus aureus: a consensus review of the microbiology, pathogenesis, and epidemiology with implications for prevention and management. American Journal of Medicine 1993, 94: 313-328.

28. Cederna JE, Terpenning MS, Ensberg M, Bradley SF, Kauffman CA: Staphylococcus aureus nasal colonization in a nursing home: cradication with mupirocin. Infection Control and Hospital Epidemiology 1990, 11: 13-16. 